\title{
Molecular confirmation of candidate Hsp70 gene associated with heat tolerance in BC3F2 advanced backcross lines and their phenotypic resemblance with recurrent chilli Kulai
}

\begin{abstract}
Domestic production of chilli in Malaysia is insufficient which accounted for $52 \%$ selfsufficiency level. Poor performance of local varieties under high temperatures above $42^{\circ} \mathrm{C}$ is one of the major factors affecting chilli production. This study was conducted with the objective of introgress heat-tolerant gene (Hsps) from AVPP0702 donor into Kulai using marker-assisted backcrossing. A total of 68 SSR markers, including Hsp linked markers that showed polymorphism between the parents were used to assess the generation of backcrossing; $\mathrm{BC} 1 \mathrm{~F} 1, \mathrm{BC} 2 \mathrm{~F} 1, \mathrm{BC} 3 \mathrm{~F} 1$ and $\mathrm{BC} 3 \mathrm{~F} 2$ and the average RPG percentage of the recurrent parent was found to be $81,90,95$ and $97 \%$, respectively. The pattern of Hsp expression in the backcross generations was similar to the donor parent (upregulated with more than 4-fold increase). Twelve improved heat tolerant chilli lines were developed. Most of the morphological and agronomical traits were recovered in the selected improved heat-tolerant genotypes from Kulai such as plant height, number of days to $50 \%$ flowering, number of fruits, fruit length and total fruit yield per plant. Improved high-yielding heat-tolerant chilli lines showed tolerance to high temperature as well as did not express any negative effect on agronomic traits in comparison with Kulai variety.
\end{abstract}

Keyword: Backcrossing; Heat-tolerant lines; Heat shock proteins; Heat stress; Marker assisted selection; Simple sequence repeat markers 